Journal of Universal Mathematics

Vol.4 No.1 PP.51-61 (2021)

ISSN-2618-5660

DOI: $10.33773 /$ jum.679843

\title{
NEW APPROACHES ON SYMMETRIC GENERALIZED INTUITIONISTIC FUZZY METRIC SPACES
}

\author{
M. JEYARAMAN AND M. SUGANTHI
}

\begin{abstract}
In this paper, we prove the existence and uniqueness of a common fixed point in symmetric generalized intuitionistic fuzzy metric spaces using property (E.A.) or CLRg property. We introduce the new notion for a pair of mappings $(f, g)$ on a generalized intuitionistic fuzzy metric space called weakly commuting of type $\left(J_{f}\right)$ and $R$-weakly commuting of type $\left(J_{f}\right)$.
\end{abstract}

\section{INTRODUCTION}

In 2006, Mustafa and Sims [9] presented a definition of $G$-metric space. After that, several fixed point results were proved in $G$-metric spaces. On the other hand, Atanassov [3] introduced the concept of intuitionistic fuzzy sets as a generalization of fuzzy sets. Park [12] has introduced and studied the notion of intuitionistic fuzzy metric spaces. In 2010, Sun and Yang [14] introduced the concept of generalized fuzzy metric space using the concept of continuous t-norm. In 2017, Muthuraj, Jeyaraman et al. [11], proved two unique common coupled fixed point theorems for Junck type and for three mappings in symmetric generalized intuitionistic fuzzy metric spaces. We prove the existence and uniqueness of a common fixed point in symmetric generalized intuitionistic fuzzy metric spaces using property (E.A.) or CLRg property. We introduce the new notion for a pair of mappings $(f, g)$ on a generalized intuitionistic fuzzy metric space called weakly commuting of type $\left(J_{f}\right)$ and $R$-weakly commuting of type $\left(J_{f}\right)$.

\section{Preliminaries}

Definition 2.1. A 5 -tuple $(X, G, H, *, \diamond)$ is said to be a generalized intuitionistic fuzzy metric space, if $X$ is an arbitrary nonempty set, $*$ is a continuous $t$-norm, $\diamond$ is a continuous $t$-conorm, $G$ and $H$ are fuzzy sets on $X^{3} \times(0, \infty)$ satisfying the following conditions:

For every $x, y, z, a \in X$ and $t, s>0$.

(i) $G(x, y, z, t)+H(x, y, z, t) \leq 1$,

(ii) $G(x, x, y, t)>0$ for $x \neq y$,

Date: January, 2020.

2000 Mathematics Subject Classification. 54H25, 47H10.

Key words and phrases. Weakly commuting of type $\left(J_{f}\right), R$-weakly commuting of type $\left(J_{f}\right)$, Generalized metric space, Intuitionistic fuzzy metric space. 
(iii) $G(x, x, y, t) \geq G(x, y, z, t)$ for $y \neq z$,

(iv) $G(x, y, z, t)=1 \quad$ if and only if $\quad x=y=z$,

(v) $G(x, y, z, t)=G(p(x, y, z), t)$, where $p$ is a permutation function,

(vi) $G(x, a, a, t) * G(a, y, z, s) \leq G(x, y, z, t+s)$,

(vii) $G(x, y, z,):.(0, \infty) \rightarrow[0,1]$ is continuous,

(viii) $G$ is a non-decreasing function on $R^{+}$,

$$
\lim _{t \rightarrow \infty} G(x, y, z, t)=1 \text { and } \lim _{t \rightarrow 0} H(x, y, z, t)=0
$$

(ix) $H(x, x, y, t)<1 \quad$ for $\quad x \neq y$,

(x) $H(x, x, y, t) \leq H(x, y, z, t)$ for $y \neq z$,

(xi) $H(x, y, z, t)=0$ if and only if $x=y=z$,

(xii) $H(x, y, z, t)=H(p(x, y, z), t)$, where $p$ is a permutation function,

(xiii) $H(x, a, a, t) \diamond H(a, y, z, s) \geq H(x, y, z, t+s)$,

(xiv) $H(x, y, z,):.(0, \infty) \rightarrow[0,1]$ is continuous,

(xv) $H$ is a non-increasing function on $R^{+}$,

$$
\lim _{t \rightarrow \infty} G(x, y, z, t)=0 \text { and } \lim _{n \rightarrow \infty} H(x, y, z, t)=1
$$

In this case, the pair $(G, H)$ is called a generalized intuitionistic fuzzy metric on $X$.

Example 2.2. Let $X$ be a nonempty set and $G$ and $H$ be metrics on $X^{3}$ where $t$-norm is defined by $a * b=\min \{a, b\}$ and $t$-conorm is defined by $a \diamond b=\max \{a, b\}$. For all $x, y, z \in X$ and $t>0$,

$$
G(x, y, z, t)=\frac{t}{t+G(x, y, z)} \quad \text { and } \quad H(x, y, z, t)=\frac{G(x, y, z)}{t+G(x, y, z)} .
$$

Then $(X, G, H, *, \diamond)$ is a generalized intuitionistic fuzzy metric space.

Definition 2.3. Let $(X, G, H, *, \diamond)$ be a generalized intuitionistic fuzzy metric space.

(i) A sequence $\left\{x_{n}\right\}$ in $X$ is said to be convergent to $x$ if

$$
\lim _{n \rightarrow \infty} G\left(x_{n}, x_{n}, x, t\right)=1 \text { and } \lim _{n \rightarrow \infty} H\left(x_{n}, x_{n}, x, t\right)=0
$$

(ii) A sequence $\left\{x_{n}\right\}$ in $X$ is said to be a Cauchy sequence if

$$
\lim _{n, m \rightarrow \infty} G\left(x_{n}, x_{n}, x_{m}, t\right)=1 \text { and } \lim _{n, m \rightarrow \infty} H\left(x_{n}, x_{n}, x_{m}, t\right)=0,
$$

that is, for any $\epsilon>0$ and for each $t>0$, there exists $n_{0} \in N$ such that

$$
G\left(x_{n}, x_{n}, x_{m}, t\right)>1-\epsilon \text { and } H\left(x_{n}, x_{n}, x_{m}, t\right)<\epsilon \text { for } n, m \geq n_{0} .
$$

(iii) A generalized intuitionistic fuzzy metric space $(X, G, H, *, \diamond)$ is said to be complete if every Cauchy sequence in $X$ is convergent.

Definition 2.4. Let $(X, G, H, *, \diamond)$ be a generalized intuitionistic fuzzy metric space. If the conditions

$$
\lim _{n \rightarrow \infty} G\left(x_{n}, y_{n}, z_{n}, t_{n}\right)=G(x, y, z, t) \quad \text { and } \quad \lim _{n \rightarrow \infty} H\left(x_{n}, y_{n}, z_{n}, t_{n}\right)=H(x, y, z, t)
$$

are satisfied whenever $\lim _{n \rightarrow \infty} x_{n}=x, \lim _{n \rightarrow \infty} y_{n}=y, \lim _{n \rightarrow \infty} z_{n}=z$ and

$$
\lim _{n \rightarrow \infty} G\left(x, y, z, t_{n}\right)=G(x, y, z, t) \quad \text { and } \quad \lim _{n \rightarrow \infty} H\left(x, y, z, t_{n}\right)=H(x, y, z, t),
$$


NEW APPROACHES ON SYMMETRIC GENERALIZED INTUITIONISTIC FUZZY METRIC53

then $G$ an d $H$ are called convergent functions on $X^{3} \times(0, \infty)$.

Lemma 2.5. Let $(X, G, H, *, \diamond)$ be a generalized intuitionistic fuzzy metric space. Then $G$ and $H$ are continuous function on $X^{3} \times(0, \infty)$.

Proof.

$$
\begin{gathered}
\text { Since } \lim _{n \rightarrow \infty} x_{n}=x, \lim _{n \rightarrow \infty} y_{n}=y, \lim _{n \rightarrow \infty} z_{n}=z \\
\text { and } \lim _{n \rightarrow \infty} G\left(x, y, z, t_{n}\right)=G(x, y, z, t), \lim _{n \rightarrow \infty} H\left(x, y, z, t_{n}\right)=H(x, y, z, t),
\end{gathered}
$$

there is $n_{0} \in N$ such that $\left|t-t_{n}\right|<\epsilon$ and $\left|t-t_{n}\right|>\delta$ for $n \geq n_{0}$ and $\epsilon<\frac{t}{2}$ and $\delta>\frac{t}{2}$.

We know that $G(x, y, z, t)$ is non-decreasing and $H(x, y, z, t)$ is non-increasing with respect to $t$. Hence we have

$$
\begin{aligned}
& G\left(x_{n}, y_{n}, z_{n}, t\right) \geq G\left(x_{n}, y_{n}, z_{n}, t-\epsilon\right) \\
& \geq G\left(x_{n}, x, x, \frac{\epsilon}{3}\right) * G\left(x, y_{n}, z_{n}, t-\frac{4 \epsilon}{3}\right) \\
& \geq G\left(x_{n}, x, x, \frac{\epsilon}{3}\right) * G\left(y_{n}, y, y, \frac{\epsilon}{3}\right) * G\left(y, x, z_{n}, t-\frac{5 \epsilon}{3}\right) \\
& \geq G\left(x_{n}, x, x, \frac{\epsilon}{3}\right) * G\left(y_{n}, y, y, \frac{\epsilon}{3}\right) * G\left(z_{n}, z, z, \frac{\epsilon}{3}\right) * G(z, y, z, t-2 \epsilon) \\
& \text { and } \\
& H\left(x_{n}, y_{n}, z_{n}, t\right) \leq H\left(x_{n}, y_{n}, z_{n}, t-\delta\right) \\
& \leq H\left(x_{n}, x, x, \frac{\delta}{3}\right) \diamond H\left(x, y_{n}, z_{n}, t-\frac{4 \delta}{3}\right) \\
& \leq H\left(x_{n}, x, x, \frac{\delta}{3}\right) \diamond H\left(y_{n}, y, y, \frac{\delta}{3}\right) \diamond H\left(y, x, z_{n}, t-\frac{5 \delta}{3}\right) \\
& \leq H\left(x_{n}, x, x, \frac{\delta}{3}\right) \diamond H\left(y_{n}, y, y, \frac{\delta}{3}\right) \diamond H\left(z_{n}, z, z, \frac{\delta}{3}\right) \diamond H(z, y, z, t-2 \delta) . \\
& G(x, y, z, t+2 \epsilon) \geq G\left(x, y, z, t_{n}+\epsilon\right) \\
& \geq G\left(x, x_{n}, x_{n}, \frac{\epsilon}{3}\right) * G\left(x_{n}, y, z, t_{n}+\frac{2 \epsilon}{3}\right) \\
& \geq G\left(x, x_{n}, x_{n}, \frac{\epsilon}{3}\right) * G\left(y, y_{n}, y_{n}, \frac{\epsilon}{3}\right) * G\left(y_{n}, x_{n}, z, t_{n}+\frac{\epsilon}{3}\right) \\
& \geq G\left(x, x_{n}, x_{n}, \frac{\epsilon}{3}\right) * G\left(y, y_{n}, y_{n}, \frac{\epsilon}{3}\right) * G\left(z, z_{n}, z_{n}, \frac{\epsilon}{3}\right) * G\left(z, y, x, t_{n}\right) \\
& \text { and } \\
& H(x, y, z, t+2 \delta) \leq H\left(x, y, z, t_{n}+\delta\right) \\
& \leq H\left(x, x_{n}, x_{n}, \frac{\delta}{3}\right) \diamond H\left(x_{n}, y, z, t_{n}+\frac{2 \delta}{3}\right) \\
& \leq H\left(x, x_{n}, x_{n}, \frac{\delta}{3}\right) \diamond H\left(y, y_{n}, y_{n}, \frac{\delta}{3}\right) \diamond H\left(y_{n}, x_{n}, z, t_{n}+\frac{\delta}{3}\right) \\
& \leq H\left(x, x_{n}, x_{n}, \frac{\delta}{3}\right) \diamond H\left(y, y_{n}, y_{n}, \frac{\delta}{3}\right) \diamond H\left(z, z_{n}, z_{n}, \frac{\delta}{3}\right) \diamond H\left(z, y, x, t_{n}\right)
\end{aligned}
$$

Let $n \rightarrow \infty$. By continuity of the functions $G$ and $H$, with respect to $t$, we can get

$$
G(x, y, z, t+2 \epsilon) \geq G(z, y, x, t) \geq G(z, y, x, t-2 \epsilon) \text { and }
$$




$$
H(x, y, z, t+2 \delta) \leq H(z, y, x, t) \leq H(z, y, x, t-2 \delta) .
$$

Therefore $G$ and $H$ are continuous functions on $X^{3} \times(0, \infty)$.

\section{Weakly Commuting of Type $\left(J_{f}\right)$}

Definition 3.1. A pair of self mapping $(f, g)$ of generalized intuitionistic fuzzy metric space $(X, G, H, *, \diamond)$ is said to be weakly commuting of type $\left(J_{f}\right)$ if

$$
G(f g x, g f x, f f x, t) \geq G(f x, g x, f x, t)
$$

and

$$
H(f g x, g f x, f f x, t) \leq H(f x, g x, f x, t)
$$

for all $x \in X$ and $t>0$.

Definition 3.2. A pair of self mapping $(f, g)$ of generalized intuitionistic fuzzy metric space $(X, G, H, *, \diamond)$ is said to be $R$-weakly commuting of type $\left(J_{f}\right)$ if there exists some positive real number $R$ such that

$$
G(f g x, g f x, f f x, t) \geq G(f x, g x, f x, t / R)
$$

and

$$
H(f g x, g f x, f f x, t) \leq H(f x, g x, f x, t / R)
$$

for all $x \in X$ and $t>0$.

Remark 3.3. If we interchange $f$ and $g$ in above definitions, the pair of self mapping $(g, f)$, of generalized intuitionistic fuzzy metric space $(X, G, H, *, \diamond)$, is said to be weekly commuting of type $\left(J_{g}\right)$ and $R$-weakly commuting of type $\left(J_{g}\right)$, respectively.

Example 3.4. Let $X=[0,1]$ be endowed with a standard generalized intuitionistic fuzzy metric defined by

$$
G(x, y, z, t)=\frac{t}{t+|x-y|+|y-z|+|z-x|}
$$

and

for all $x, y, z \in X$ and $t>0$.

$$
H(x, y, z, t)=\frac{|x-y|+|y-z|+|z-x|}{t+|x-y|+|y-z|+|z-x|}
$$

Define $f, g: X \rightarrow X$ by $f x=\frac{x^{2}}{4}, g x=x^{2}$, for all $x \in X$. Clearly, $x=0$ is the only coincidence point of $f$ and $g$.

So $f$ and $g$ are weakly compatible. It should be noted that

$$
G(f g x, g f x, f f x, t) \geq G(f x, g x, f x, t), H(f g x, g f x, f f x, t) \leq H(f x, g x, f x, t)
$$

for all $x \in X$ and $t>0$.

Then the pair $(f, g)$ is weakly commuting of type $\left(J_{f}\right)$ but not weakly commuting of type $\left(J_{g}\right)$.

Example 3.5. Let $X=[0,2]$ be endowed with a standard generalized intuitionistic fuzzy metric. Define $f x=2-x, g x=x$, then by an easy calculation, one can show that the pair $(f, g)$ is weakly commuting of type $\left(J_{f}\right)$ and $R$-weakly commuting of type $\left(J_{f}\right)$. 
Lemma 3.6. If $f$ and $g$ are weakly commuting of type $\left(J_{f}\right)$ or $R$-weakly commuting of type $\left(J_{f}\right)$, then $f$ and $g$ are weakly compatible.

Proof. Let $x$ be a coincidence point of $f$ and $g$, i.e., $f x=g x$. If the pair $(f, g)$ of generalized intuitionistic fuzzy metric space $(X, G, H, *, \diamond)$ is weakly commuting of type $\left(J_{f}\right)$, we have

$$
G(f g x, g f x, f g x, t)=G(f g x, g f x, f f x, t) \geq G(f x, g x, g x, t) \geq 1,
$$

and

$$
H(f g x, g f x, f g x, t)=H(f g x, g f x, f f x, t) \leq H(f x, g x, g x, t) \leq 0 .
$$

It follows that $f g x=g f x$, and then they commute at their coincidence point. Similarly, if the pair $(f, g)$ of generalized intuitionistic fuzzy metric space $(X, G, H, *, \diamond)$ is $R$-weakly commuting of type $\left(J_{f}\right)$, we have, for all $x \in X$,

$$
\begin{aligned}
& G(f g x, g f x, f g x, t) \geq G(f g x, g f x, f f x, t) \geq G(f x, g x, f x, t / R)=1, \\
& H(f g x, g f x, f g x, t) \leq H(f g x, g f x, f f x, t) \leq H(f x, g x, f x, t / R)=0 .
\end{aligned}
$$

Thus $f g x=g f x$, which implies that $f$ and $g$ are weakly compatible.

Definition 3.7. [1] A pair of self mapping $(f, g)$ on $X$ is said to satisfy the property (E.A.) if there exists a sequence $\left\{x_{n}\right\}$ such that

$$
\lim _{n \rightarrow \infty} f x_{n}=\lim _{n \rightarrow \infty} g x_{n}=z \text { for all } z \in X
$$

Definition 3.8. A pair of self mapping $(f, g)$ on $X$ is said to satisfy CLRg property if there exists a sequence $\left\{x_{n}\right\}$ such that

$$
\lim _{n \rightarrow \infty} f x_{n}=\lim _{n \rightarrow \infty} g x_{n}=g z \text { for all } z \in X .
$$

For proving our main results, we use the following relation:

Define $\Phi=\left\{\phi: \mathbb{R}^{+} \rightarrow \mathbb{R}^{+}\right\}$where $\mathbb{R}^{+}=[0, \infty)$ and each $\phi \in \Phi$ satisfies the following conditions:

$(\Phi-1): \phi$ is strictly increasing.

$(\Phi-2): \phi$ is upper semi-continuous from the right.

$(\Phi-3): \sum_{n=0}^{\infty} \phi^{n}(t)<\infty$ for all $t>0$.

Lemma 3.9. Let $(X, G, H, *, \diamond)$ be a generalized intuitionistic fuzzy metric space. If there exists $\phi \in \Phi$ such that

$$
G(x, y, z, \phi(t)) \geq G(x, y, z, t) \text { and } H(x, y, z, \phi(t)) \leq H(x, y, z, t) \text { for all } t>0,
$$

then $x=y=z$.

Lemma 3.10. Let $(X, G, H, *, \diamond)$ be a generalized intuitionistic fuzzy metric space and $\left\{y_{n}\right\}$ be a sequence in $X$. If there exists $\phi \in \Phi$ such that

$$
G\left(y_{n}, y_{n}, y_{n+1}, \phi(t)\right) \geq G\left(y_{n-1}, y_{n-1}, y_{n}, t\right) * G\left(y_{n}, y_{n}, y_{n+1}, t\right)
$$

and

$$
H\left(y_{n}, y_{n}, y_{n+1}, \phi(t)\right) \leq H\left(y_{n-1}, y_{n-1}, y_{n}, t\right) \diamond H\left(y_{n}, y_{n}, y_{n+1}, t\right)
$$

for all $t>0$ and $n=1,2, \ldots$,

then $\left\{y_{n}\right\}$ is a Cauchy sequence in $X$. 


\section{Main Results}

Theorem 4.1. Let $(X, G, H, *, \diamond)$ be a symmetric generalized intuitionistic fuzzy metric space and the mappings $f, g: X \rightarrow X$ satisfy the following conditions: (4.1.1) $f$ and $g$ are weakly commuting of type $\left(J_{f}\right)$,

(4.1.2) $f(X) \subseteq g(X)$

(4.1.3) $g(X)$ is a complete subspace of $X$,

(4.1.4) there exists $a \phi \in \Phi$ such that for all $x, y, z \in X$ and $t>0$,

$$
\begin{aligned}
G(f x, f y, f z, \phi(t)) & \geq G(g x, g x, f x, t) * G(g y, g y, f y, t) * G(g z, g z, f z, t), \\
H(f x, f y, f z, \phi(t)) & \leq H(g x, g x, f x, t) \diamond H(g y, g y, f y, t) \diamond H(g z, g z, f z, t) .
\end{aligned}
$$

Then $f$ and $g$ have common fixed point.

Proof. Let $x_{0}, x_{1}, x_{2} \in X$ be such that $f x_{0}=g x_{1}$ and $f x_{1}=g x_{2}$. Then by induction, we can define a sequence $\left\{y_{n}\right\} \in X$ as follows:

$$
y_{n}=f x_{n}=g x_{n+1}, n \in N \text {. }
$$

We will prove $\left\{y_{n}\right\}$ is a Cauchy sequence in $X$.

$$
\begin{aligned}
& G\left(y_{n}, y_{n}, y_{n+1}, \phi(t)\right)=G\left(f x_{n}, f x_{n}, f x_{n+1}, \phi(t)\right) \\
& \geq G\left(g x_{n}, g x_{n}, f x_{n}, t\right) * G\left(g x_{n}, g x_{n}, f x_{n}, t\right) * G\left(g x_{n+1}, g x_{n+1}, f x_{n+1}, t\right) \\
& \geq G\left(g x_{n}, g x_{n}, g x_{n+1}, t\right) * G\left(g x_{n}, g x_{n}, g x_{n+1}, t\right) * G\left(g x_{n+1}, g x_{n+1}, g x_{n+2}, t\right) \\
& =G\left(g x_{n}, g x_{n}, g x_{n+1}, t\right) * G\left(g x_{n+1}, g x_{n+1}, g x_{n+2}, t\right) \quad \text { and } \\
& H\left(y_{n}, y_{n}, y_{n+1}, \phi(t)\right)=H\left(f x_{n}, f x_{n}, f x_{n+1}, \phi(t)\right) \\
& \leq H\left(g x_{n}, g x_{n}, f x_{n}, t\right) \diamond H\left(g x_{n}, g x_{n}, f x_{n}, t\right) \diamond H\left(g x_{n+1}, g x_{n+1}, f x_{n+1}, t\right) \\
& \leq H\left(g x_{n}, g x_{n}, g x_{n+1}, t\right) \diamond H\left(g x_{n}, g x_{n}, g x_{n+1}, t\right) \diamond H\left(g x_{n+1}, g x_{n+1}, g x_{n+2}, t\right) \\
& =H\left(g x_{n}, g x_{n}, g x_{n+1}, t\right) \diamond H\left(g x_{n+1}, g x_{n+1}, g x_{n+2}, t\right) .
\end{aligned}
$$

This gives

$$
G\left(y_{n}, y_{n}, y_{n+1}, \phi(t)\right) \geq G\left(y_{n-1}, y_{n-1}, y_{n}, t\right) * G\left(y_{n}, y_{n}, y_{n+1}, t\right)
$$

and

$$
H\left(y_{n}, y_{n}, y_{n+1}, \phi(t)\right) \leq H\left(y_{n-1}, y_{n-1}, y_{n}, t\right) \diamond H\left(y_{n}, y_{n}, y_{n+1}, t\right) .
$$

By the lemma (3.10), the sequence $\left\{y_{n}\right\}$ is a Cauchy sequence.

Since $y_{n}=g x_{n+1},\left\{g x_{n+1}\right\}$ is a Cauchy sequence in $g(X)$.

By (4.1.3) hypotheses, we know that

$g(X)$ is complete and then there exists $u \in g(X)$ such that

$$
\lim _{n \rightarrow \infty} g x_{n}=u=\lim _{n \rightarrow \infty} f x_{n} .
$$

Now $u \in g(X)$, so there exists $p \in X$ such that $u=g p$.

Therefore

We will prove that $f p=g p$.

$$
\lim _{n \rightarrow \infty} g x_{n}=g p=\lim _{n \rightarrow \infty} f x_{n}
$$

$G\left(f p, f p, f x_{n}, \phi(t)\right) \geq G(g p, g p, f p, t) * G(g p, g p, f p, t) * G\left(g x_{n}, g x_{n}, f x_{n}, t\right)$ and

$$
H\left(f p, f p, f x_{n}, \phi(t)\right) \leq H(g p, g p, f p, t) \diamond H(g p, g p, f p, t) \diamond H\left(g x_{n}, g x_{n}, f x_{n}, t\right) .
$$

Taking limit as $n \rightarrow \infty$, we have

$$
G(f p, f p, g p, \phi(t)) \geq G(g p, g p, f p, t) * G(g p, g p, f p, t) * G(g p, g p, f p, t)
$$


or

$$
\begin{gathered}
G(f p, f p, g p, \phi(t)) \geq G(g p, g p, f p, t) \text { and } \\
H(f p, f p, g p, \phi(t)) \leq H(g p, g p, f p, t) \diamond H(g p, g p, f p, t) \diamond H(g p, g p, g p, t)
\end{gathered}
$$

or

$$
H(f p, f p, g p, \phi(t)) \leq H(g p, g p, f p, t) .
$$

Since generalized intuitionistic fuzzy metric space is symmetric, we have

$$
\begin{aligned}
& G(f p, f p, g p, \phi(t)) \geq G(g p, g p, f p, t)=G(f p, f p, g p, t), \\
& H(f p, f p, g p, \phi(t)) \leq H(g p, g p, f p, t)=H(f p, f p, g p, t)
\end{aligned}
$$

which implies $f p=g p$ (by Lemma (3.9)).

Since the pair $(f, g)$ is weakly commuting of type $\left(J_{f}\right)$, then

$$
G(f g p, g f p, f f p, \phi(t)) \geq G(f p, g p, f p, t)=1
$$

and

$$
H(f g p, g f p, f f p, \phi(t)) \leq H(f p, g p, f p, t)=0,
$$

implies that $f f p=f g p=g f p=g g p$. Hence $f u=f g p=g f p=g u$.

Finally, we show that $u=g p$ is common fixed point of $f$ and $g$.

Suppose $f u \neq u$, then

$$
\begin{aligned}
G(f u, f p, f p, \phi(t)) & \geq G(g u, g u, f u, t) * G(g p, g p, f p, t) * G(g p, g p, f p, t), \\
G(f u, f p, f p, \phi(t)) & \geq G(f u, f u, f u, t) * G(f p, f p, f p, t) * G(f p, f p, f p, t), \\
G(f u, u, u, \phi(t)) & \geq 1 * 1 * 1=1, \\
H(f u, f p, f p, \phi(t)) & \leq H(g u, g u, f u, t) \diamond H(g p, g p, f p, t) \diamond H(g p, g p, f p, t), \\
H(f u, f p, f p, \phi(t)) & \leq H(f u, f u, f u, t) \diamond H(f p, f p, f p, t) \diamond H(f p, f p, f p, t), \\
H(f u, u, u, \phi(t)) & \leq 0 \diamond 0 \diamond 0=0, \text { a contradiction }
\end{aligned}
$$

Hence $f u=g u=u$.

To prove the uniqueness, suppose we have $u$ and $\mathrm{v}$ such that $\mathrm{u} \neq v$ and

$$
\mathrm{fu}=\mathrm{gu}=\mathrm{u} \quad \text { and } \quad \mathrm{fv}=\mathrm{gv}=\mathrm{v}
$$

Using condition (4.1.4), we have

$$
\begin{aligned}
G(u, v, v, \phi(t)) & =G(f u, f v, f v, \phi(t)) \\
& \geq G(g u, g u, f u, t) * G(g v, g v, f v, t) * G(g v, g v, f v, t) \\
& =1 * 1 * 1=1, \\
H(u, v, v, \phi(t)) & =H(f u, f v, f v, \phi(t)) \\
& \leq H(g u, g u, f u, t) \diamond H(g v, g v, f v, t) \diamond H(g v, g v, f v, t) \\
& =0 \diamond 0 \diamond 0=0 .
\end{aligned}
$$

$$
\text { Hence } G(u, v, v, \phi(t)) \geq 1 \quad \text { and } \quad H(u, v, v, \phi(t)) \leq 0 \text {, }
$$

which gives contradiction. Hence $u=v$. Therefore $u$ is a unique common fixed point. 
Example 4.2. Let $X=[0,1]$ be endowed with standard generalized intuitionistic fuzzy metric. Define $f, g: X \rightarrow X$ by $f(x)=\frac{x^{2}}{4}, g(x)=x^{2}, x \in X$. We see that $x=0$ is the only coincidence point.

So $f$ and $g$ are weakly compatible.

$$
\begin{aligned}
& \text { Also } G(f g x, g f x, f f x, t) \geq G(f x, g x, f x, t) \\
& \text { and } H(f g x, g f x, f f x, t) \leq H(f x, g x, f x, t) .
\end{aligned}
$$

Then the pair $(f, g)$ is weakly commuting of type $\left(J_{f}\right)$ but not weakly commuting of type $\left(J_{g}\right)$.

Example 4.3. Let $X=[-1,1]$ be endowed with standard generalized intuitionistic fuzzy metric. Let $\phi(t)=\frac{t}{2}$ and define $f, g: X \rightarrow X$ by $f(x)=\frac{x}{6}, g(x)=\frac{x}{2}(x+1)$, $x \in X$. We see that $x=0$ is the only coincidence point and $f$ and $g$ are weakly compatible.

Let $\left\{x_{n}=\frac{1}{n}\right\}$ be a sequence such that

$$
G\left(f p, f p, f x_{n}, \phi(t)\right) \geq G(f p, f p, g p, t)
$$

and

$$
H\left(f p, f p, f x_{n}, \phi(t)\right) \leq H(f p, f p, g p, t)
$$

where $p$ is a coincidence point.

Then the pair $(f, g)$ is weakly commuting of type $\left(J_{f}\right)$ and $f$ and $g$ have a unique common fixed point.

Corollory 4.4. Theorem (4.1) remains true if we replace weakly commuting of type $\left(J_{f}\right)$ by weakly commuting of type $\left(J_{g}\right)$ or replace $R$-weakly commuting of type $\left(J_{g}\right)$.

Theorem 4.5. Let $(X, G, H, *, \diamond)$ be a symmetric generalized intuitionistic fuzzy metric space and suppose mappings $f, g: X \rightarrow X$ are weakly commuting of type $\left(J_{f}\right)$ satisfying the following conditions:

(4.5.1) $f$ and $g$ satisfy the property (E.A.),

(4.5.2) $g(X)$ is a closed subspace of $X$,

(4.5.3) there exists $a \phi \in \Phi$ such that for all $x, y, z \in X$ and $t>0$,

$$
G(f x, f y, f z, \phi(t)) \geq G(g x, g x, f x, t) * G(g y, g y, f y, t) * G(g z, g z, f z, t)
$$

and

$$
H(f x, f y, f z, \phi(t)) \leq H(g x, g x, f x, t) \diamond H(g y, g y, f y, t) \diamond H(g z, g z, f z, t) .
$$

Then $f$ and $g$ have a unique common fixed point.

Proof. The mappings $f$ and $g$ satisfy the property (E.A.), then there exists a sequence $\left\{x_{n}\right\} \in X$ satisfying

$$
\lim _{n \rightarrow \infty} f x_{n}=u=\lim _{n \rightarrow \infty} g x_{n}
$$

for some $u \in X$.

Since $g(X)$ is a closed subspace of $X$ and

$$
\lim _{n \rightarrow \infty} g x_{n}=u
$$

then there exists $p \in X$ such that $g p=u$.

$$
\text { Also } \lim _{n \rightarrow \infty} g x_{n}=g p=\lim _{n \rightarrow \infty} f x_{n}
$$


We will prove, $f p=g p$.

$$
G\left(f p, f p, f x_{n}, \phi(t)\right) \geq G(g p, g p, f p, t) * G(g p, g p, f p, t) * G\left(g x_{n}, g x_{n}, f x_{n}, t\right)
$$

and

$$
H\left(f p, f p, f x_{n}, \phi(t)\right) \leq H(g p, g p, f p, t) \diamond H(g p, g p, f p, t) \diamond H\left(g x_{n}, g x_{n}, f x_{n}, t\right),
$$

taking limit as $n \rightarrow \infty$, we have

$$
\begin{aligned}
& G(f p, f p, g p, \phi(t)) \geq G(g p, g p, f p, t) * G(g p, g p, f p, t) * G(g p, g p, f p, t) \\
& G(f p, f p, g p, \phi(t)) \geq G(g p, g p, f p, t), \text { and } \\
& H(f p, f p, g p, \phi(t)) \leq H(g p, g p, f p, t) \diamond H(g p, g p, f p, t) \diamond H(g p, g p, f p, t) \\
& H(f p, f p, g p, \phi(t)) \leq H(g p, g p, f p, t) .
\end{aligned}
$$

Since generalized intuitionistic fuzzy metric space is symmetric, we have

$$
G(f p, f p, g p, \phi(t)) \geq G(f p, f p, g p, t)
$$

and

$$
H(f p, f p, g p, \phi(t)) \leq H(f p, f p, g p, t),
$$

which implies $f p=g p=u$.

Since the pair $(f, g)$ is weakly commuting of type $\left(J_{f}\right)$, then

$$
G(f g p, g f p, f f p, \phi(t)) \geq G(f p, g p, f p, t)=1
$$

and

$$
H(f g p, g f p, f f p, \phi(t)) \leq H(f p, g p, f p, t)=0,
$$

which implies $f f p=f g p=g f p=g g p$. Hence $f u=f g p=g f p=g u$.

Now, we will show that $f p=u$ is a common fixed point of $f$ and $g$.

Suppose $f u \neq u$, then

$$
\begin{aligned}
G(f u, u, u, \phi(t)) & =G(f u, f p, f p, \phi(t)) \\
& \geq G(g u, g u, f u, t) * G(g p, g p, f p, t) * G(g p, g p, f p, t) \\
& =G(f u, f u, f u, t) * 1 * 1 \\
& =1 * 1 * 1=1, \\
H(f u, u, u, \phi(t)) & =H(f u, f p, f p, \phi(t)) \\
& \leq H(g u, g u, f u, t) \diamond H(g p, g p, f p, t) \diamond H(g p, g p, f p, t) \\
& =H(f u, f u, f u, t) \diamond 0 \diamond 0 \\
& =0 \diamond 0 \diamond 0=0,
\end{aligned}
$$

a contradiction. Hence $f u=u=g u$.

To prove the uniqueness, suppose we have $u$ and $v$ such that $u \neq v, f u=g u=u$ and $f v=g v=v$, then again using condition (4.5.3), we have,

$$
\begin{aligned}
G(u, v, v, \phi(t)) & =G(f u, f v, f v, \phi(t)) \\
& \geq G(g u, g u, f u, t) * G(g v, g v, f v, t) * G(g v, g v, f v, t) \\
& =1 * 1 * 1=1, \\
H(u, v, v, \phi(t)) & =H(f u, f v, f v, \phi(t)) \\
& \leq H(g u, g u, f u, t) \diamond H(g v, g v, f v, t) \diamond H(g v, g v, f v, t) \\
& =0 \diamond 0 \diamond 0=0,
\end{aligned}
$$


which is a contradiction. Hence $u=v$. Therefore $u$ is a unique common fixed point of $f$ and $g$.

Theorem 4.6. Let $(X, G, H, *, \diamond)$ be a symmetric generalized intuitionistic fuzzy metric space and suppose mappings $f, g: X \rightarrow X$ are weakly commuting of type $\left(J_{f}\right)$ satisfying the following conditions:

(4.6.1) $f$ and $g$ satisfy CLRg property,

(4.6.2) there exists a $\phi \in \Phi$ such that for all $x, y, z \in X$ and $t>0$,

$$
\begin{gathered}
G(f x, f y, f z, \phi(t)) \geq G(g x, g x, f x, t) * G(g y, g y, f y, t) * G(g z, g z, f z, t) \\
H(f x, f y, f z, \phi(t)) \leq H(g x, g x, f x, t) \diamond H(g y, g y, f y, t) \diamond H(g z, g z, f z, t) .
\end{gathered}
$$

Then $f$ and $g$ have a unique common fixed point.

Proof. The proof follows on the same lines of Theorem(4.5) and by definition of CLRg property.

\section{CONCLUSION}

This paper introduced new kinds of pair of self mappings viz. weakly commuting of type $\left(J_{f}\right)$ and R-weakly commuting of type $\left(J_{f}\right)$ on generalized intuitionistic fuzzy metric spaces. Common fixed point theorems are proved based on these newly introduced pair of self mappings. This work provides a new approach to analyze intuitionistic fuzzy metric spaces and paves a path that leads to extensions of such conceptual work.

\section{REFERENCES}

[1] M. Aamri and D. El. Moutawakil, Some new common fixed point theorems under strict contractive conditions", Math. Anal. Appl. 270 (2002), 181 - 188.

[2] C. Alaca, D. Turkoglu and C. Yildiz, Fixed points in intuitionistic fuzzy metric space, Chaos, Soliton and Fractals, 29 (5) (2006), 1073 - 1078.

[3] K. Atanassov, Intuitionistic fuzzy sets, Fuzzy Sets and System, 20 (1986), 87 - 96.

[4] A. George and P. Veeramani, On some results in fuzzy metric spaces, Fuzzy Sets Syst. 64 (1994), 395 - 399.

[5] A. George and P. Veeramani, On some results of analysis for fuzzy metric spaces, Fuzzy Sets Syst. 90 (1997), 365 - 368.

[6] G. Jungck, Commuting mappings and fixed points, Am. Math. Mon. 83 (1976),261 - 263.

[7] O. Kramosil and J. Michalek, Fuzzy metric and statistical metric spaces, Kybernetica 11 (1975), 326 - 334.

[8] S. Manro, S. S. Bhatia and S. Kumar, Common fixed point theorems in fuzzy metric spaces, Annals of Fuzzy Mathematics and Informatics, 3(1) (2012), 151 - 158.

[9] Z. Mustafa and B. Sims, A new approach to generalized metric spaces, J. Nonlinear Convex Anal. 7 (2006), 289 - 297.

[10] Z. Mustafa, H. Aydi and E. Karapinar, On common fixed points $G$ - metric spaces using (E.A.) property, Computer and mathematics with applications 64 (2012), 1944 - 1956.

[11] R. Muthuraj, M. Sornavalli and M. Jeyaraman, Common coupled fixed point theorems in generalized intuitionistic fuzzy metric spaces, Notes On Intuitionistic Fuzzy sets, 23(1) (2017), $57-69$

[12] J. H. Park, Intuitionistic fuzzy metric space, Chaos, Solitions \& Fractals, 22 (2004), 1039 1046.

[13] S. Sessa, On a weak commutativity condition of mappings in fixed point considerations, Publ. Inst. Math. 32 (46) (1982), 149 - 153.

[14] G. Sun and K. Yang, Generalized fuzzy metric spaces with properties, Res. J. Appl. Sci. 2 (2010), $673-678$.

[15] L. A. Zadeh, Fuzzy sets, Inf. Control 8 (1965), 338 - 353. 
NEW APPROACHES ON SYMMETRIC GENERALIZED INTUITIONISTIC FUZZY METRIC61

Department of Mathematics, Raja Doraisingam Government Arts College, SivaganGai - 630561, Affiliated to Alagappa University, Karaikudi, Tamil Nadu, India

Email address: jeya.math@gmail.com

Research Scholar, Department of Mathematics, Raja Doraisingam Government Arts College, Sivagangai - 630561, Affiliated to Alagappa University, Karaikudi, Tamil NADU, INDIA.

Email address: vimalsugan@gmail.com 